\title{
Aspectos psicosociales en pacientes candidatos a trasplante de órganos
}

\section{Psychosocial aspects in candidates for organ transplant}

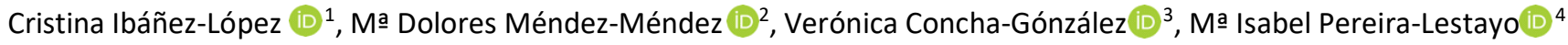 \\ 1. Hospital Universitario Marqués de Valdecilla. Santander, España. Correo: ibanezlopezcristina@gmail.com - https://orcid.org/0000-0002-0529-7443 \\ 2. Hospital Universitario Central de Asturias. Oviedo, España. Correo: Immendezloli@gmail.com - https://orcid.org/0000-0001-5535-113X \\ 3. Hospital Universitario Central de Asturias. Oviedo, España. Correo: veronicaconcha89@gmail.com - https://orcid.org/0000-0002-7644-7242 \\ 4. Hospital del Oriente de Asturias Francisco Grande Covián. Arriondas, España. Correo: isaples@gmail.com - https://orcid.org/0000-0002-0494-766X
}

Tipología: Artículo de investigación científica y tecnológica

Para citar este artículo: Ibáñez-López C, Méndez-Méndez MD, Concha-González V, Pereira-Lestayo MI. Aspectos psicosociales en pacientes candidatos a trasplante de órganos. Duazary. 2021 enero; 18(1): 20-31. Doi: https://doi.org/10.21676/2389783X.3886

Recibido en octubre 30 de 2019

Aceptado en julio 31 de 2020

Publicado en línea en enero 07 de 2021

Palabras
clave:
trasplante;
psicología
clínica; calidad
de vida;
enfermedad
crónica.

Palabras

clave:

trasplante

psicología

clínica; calidad

enfermedad

crónica.

\section{RESUMEN}

Este estudio analiza las variables psicosociales implicadas en el proceso de trasplante de órganos y plantea las posibles contribuciones de la psicología clínica en su abordaje. Se trata de un estudio descriptivo-transversal con muestra no probabilística intencional de $\mathrm{N}=64$ (51 hombres y 13 mujeres), con edades comprendidas entre 37 y 66 años (Media=58,17; Desviación Típica=6,038). La evaluación se realiza mediante la entrevista clínica, la aplicación de la Escala de Ansiedad y Depresión Hospitalaria de Zimong y Shaith y del Mini-Examen del Estado Mental de Folstein y colaboradores. Se encuentra que entre el $56-68 \%$ de los pacientes tiene conciencia de la necesidad de mantener hábitos saludables. Un $37,5 \%$ del grupo de trasplante hepático no cumple abstinencia alcohólica superior a 6 meses. Más de la mitad de los candidatos tienen antecedentes en salud mental y aproximadamente el $20 \%$ presenta sintomatología clínica. Entre el $62-81 \%$ está motivado para la intervención. La influencia de los factores psicosociales en la evolución y el pronóstico de los trasplantes muestra la necesidad de fomentar la participación del psicólogo clínico en la evaluación y en el desarrollo de intervenciones adecuadas a las necesidades específicas de cada fase, favoreciendo la atención sanitaria integral durante todo el proceso.

\section{ABSTRACT}

Keywords: Organ Transplantati on;

Psychology

Clinical; Quality of Life; Chronic Disease.
This study analyzes the psychosocial variables involved in the organ transplant process and presents the possible contributions of clinical psychology in its approach. That is a descriptive cross-sectional study with an intentional non-probabilistic sample of $\mathrm{N}=64$ (51 men and 13 women), with ages between 37 and 66 years (Mean=58.17; Standard Deviation=6.038). The evaluation is carried out through clinical interviews, the application of the Zimong and Shaith Hospital Anxiety and Depression Scale and the Folstein et al. Mini-Exam of Mental State. Results show that between 56-68\% of patients are aware of the need to maintain healthy habits. Also, $37.5 \%$ of the liver transplant group do not comply with alcohol abstinence for more than six months. More than half of the candidates have a mental health history and approximately $20 \%$ have clinical symptoms. Between $62-81 \%$ are motivated by the intervention. The influence of psychosocial factors on the evolution and prognosis of transplants shows the need to encourage the participation of the clinical psychologist in the assessment and development of interventions appropriate to the specific needs of each phase, favoring comprehensive sanitary care throughout the process. 


\section{INTRODUCCIÓN}

La cirugía de trasplante conforma uno de los grandes avances médicos de los últimos tiempos, dado su papel en la recuperación de salud y en el fomento de la calidad de vida en personas que padecen enfermedades crónicas ${ }^{1-4}$.

En 1965 se realizó el primer trasplante en España en el Hospital Clínico de Barcelona. Consistió en un trasplante renal a una mujer de 35 años. Han pasado más de 50 años y el número de intervenciones destinadas a la donación y trasplante de órganos se ha incrementado notablemente debido a sus resultados y al aumento de las patologías de carácter crónico, que son actualmente un importante problema para la salud pública ${ }^{5,6}$.

El modelo español de donación y trasplante de órganos, basado en los principios de universalidad y donación anónima, se posiciona como un referente a nivel mundial7,8. La Organización Mundial de la Salud y la Unión Europea ${ }^{7}$ resaltan su alta tasa de donantes (36 donantes por millón de población) y su eficacia. Sus resultados se han traducido, por un lado, en un aumento de la esperanza y la calidad de vida y, por otro, en la reducción de la tasa de mortalidad poblacional ${ }^{9-11}$. Según los datos de la Organización Nacional de Trasplantes, en el año 2016 se realizaron en España 4821 trasplantes de órganos sólidos: 2997 renales, 1159 hepáticos, 307 pulmonares, 281 cardíacos, 73 de páncreas y 4 intestinales. Este mismo año, en el Hospital Universitario Central de Asturias (HUCA) se realizaron 111 intervenciones: 57 renales, 40 hepáticas, 14 cardiacas y 12 derivaciones a pulmonar $^{8}$.

El trasplante de órganos requiere un abordaje de carácter multidisciplinar ${ }^{5,12}$, puesto que el proceso de adaptación a una enfermedad crónica y su tratamiento, de carácter no curativo, es un fenómeno que trasciende a la patología médica en sí. Implica una serie de cambios que afectan a diversas esferas de la vida de la persona, incluidas la psicológica y la social ${ }^{5,12-14}$. El área emocional es una de las especialmente afectadas y provoca gran interferencia en el bienestar del paciente, dado el complejo proceso al que necesariamente se ve sometida la persona candidata a trasplante ${ }^{4,15}$. En ocasiones supone la sustitución de un problema médico por otro ${ }^{16}$. Entre los posibles problemas psicosociales de los receptores de trasplantes se incluyen diagnósticos psiquiátricos, reacciones emocionales secundarias a las dificultades en el ajuste individual y familiar, disfunciones sexuales, problemas en la adherencia a los tratamientos, bajo índice de retorno a la actividad laboral y deshabituación de consumo de tóxicos ${ }^{13,14,17-19}$. Es necesario dirigir las intervenciones a cada una de estas áreas problemáticas, ya que el propósito último del trasplante de órganos es la mejora en la calidad de vida de la persona, ${ }^{5,20}$, más allá de la supervivencia del paciente.

Dentro del equipo de trabajo interdisciplinar, la figura del psicólogo clínico cumple un importante papel en la atención a pacientes y familiares que experimentan el proceso de recepción de órganos ${ }^{21,22}$. Esta función trasciende la evaluación y se inicia en el preciso instante en el que el paciente conoce sus opciones de tratamiento ${ }^{23}$. El candidato requiere una preparación psicológica para ser trasplantado, tornándose imprescindible una completa evaluación de carácter biopsicosocial de los factores que pudieran afectar al proceso ${ }^{17}$. Variables como el estado anímico, el estilo de vida y el apoyo psicosocial deben ser especialmente recogidas y atendidas, dada su relevancia para la recuperación ${ }^{24}$. Estas, afectan a la adaptación postquirúrgica e influyen en la capacidad para mantener un estilo de vida saludable, la comunicación con el médico y las habilidades de autocuidado. Se ha encontrado que las dificultades en la asimilación de la nueva situación vital, las expectativas de vida o la dificultad en la consecución de las metas a medio-largo plazo, favorecen la aparición de sintomatología psíquica, siendo la ansiedad, depresión, intencionalidad suicida y psicosis las más comunes ${ }^{13}$.

Según algunos autores, en la intervención del psicólogo clínico pueden distinguirse cuatro momentos diferentes: evaluación psicosocial, acompañamiento y seguimiento prequirúrgico, postrasplante inmediato y postrasplante tardío ${ }^{25}$. Su 
labor comienza en el proceso de evaluación clínica, en el que expone los criterios que valoran la idoneidad de la realización del trasplante, modula la comunicación con el equipo interdisciplinario en la toma de decisiones y facilita herramientas a los candidatos a trasplante y sus familias para una mejora en su estilo de vida, el afrontamiento de su enfermedad y la adherencia a los tratamientos requeridos ${ }^{14,21,26}$.

El objetivo, en definitiva, es facilitar la adaptación del paciente y su familia a la nueva etapa que les corresponde vivir $^{21}$, mediante la cobertura asistencial de sus necesidades médicas, psicológicas y psicosociales específicas. En este sentido, se recomienda realizar seguimientos periódicos en el momento previo y posterior a la intervención ${ }^{23}$.

Conforme a la literatura, la evaluación psicosocial busca principalmente identificar los recursos de afrontamiento de las condiciones posteriores al trasplante ${ }^{4,5,23}$, la existencia de capacidad y calidad del apoyo de la red social y el abordaje de aquellos factores que contribuyen a incrementar la probabilidad de éxito de la intervención ${ }^{19,20,25}$. Concretamente, la valoración psicológica debe incidir en los siguientes aspectos: el deseo del paciente de ser trasplantado, la calidad y la cantidad de soporte sociofamiliar, las expectativas sobre la intervención propuesta, la capacidad de comprensión de los riesgos y beneficios asociados a la intervención, la capacidad para cumplir el tratamiento y el propósito veraz de la persona de establecer un cambio en su estilo de vida ${ }^{14,19,27}$.

De acuerdo con algunos estudios, en la fase de evaluación previa al trasplante, un porcentaje importante de pacientes son rechazados por factores psicosociales como enfermedades mentales no tratadas, consumo activo de alcohol $u$ otras sustancias tóxicas, o no disponer de un cuidador $^{28}$. No existe un protocolo unificado de evaluación entre los diferentes hospitales, con la consiguiente limitación en la selección idónea de los candidatos $^{28}$.

A la luz de los datos, se hace patente la importancia de desarrollar programas interdisciplinares específicos que incluyan la figura del psicólogo clínico en el desarrollo de la evaluación e intervención psicoeducativa y psicoterapéutica ${ }^{21,27}$. Son necesarios abordajes que, además de la elaboración de un protocolo de actuación, incidan en las necesidades concretas de esta población y mejoren la eficiencia, la eficacia y la efectividad de nuestro sistema sanitario y, por ende, la calidad asistencial proporcionada a los usuarios.

Con base en lo expuesto, el objetivo principal del presente estudio es conocer las variables psicosociales implicadas en el abordaje del proceso de trasplante de órganos, reflexionar sobre el rol del psicólogo clínico en estos equipos interdisciplinares y considerar posibles actuaciones de mejora en la valoración psicológica y la intervención de estos pacientes.

Para tal fin, se recurre al análisis del perfil sociodemográfico y sociofamiliar de los candidatos a trasplante, a la exploración de los hábitos de salud y del estado clínico de los pacientes, teniendo en cuenta posibles alteraciones anímicas asociadas y dificultades de afrontamiento. Asimismo, se ahonda en los factores intrínsecos al proceso, tales como información disponible y motivación para someterse a la intervención.

\section{MATERIALES Y MÉTODOS}

\section{Tipo de estudio}

Estudio descriptivo cuantitativo de carácter transversal ${ }^{29}$.

\section{Población y muestra}

La población objeto de estudio son las personas candidatas a trasplante. La muestra se compuso por los pacientes pulmonares y hepáticos derivados del Programa de Valoración Psicológica del Servicio de Salud Mental de Enlace e Interconsulta del Hospital Universitario Central de Asturias - HUCA. Se excluyeron los candidatos a trasplante renal por tratarse de una muestra no representativa $(n=3)$.

Los resultados de este artículo se corresponden a los datos acopiados en las evaluaciones psicológicas realizadas entre los meses de enero y diciembre de 
2017. La muestra total ( $N=64)$ se compone de un $79,7 \%$ de hombres y un $20,3 \%$ de mujeres. La edad media $(X)$ es de 58,17 años, con una Desviación Típica (DT) de 6,03.

Respecto al tipo de trasplante, el $75 \%$ fue valorado para Trasplante de Hígado (TH) y el $25 \%$ restante para valoración de Trasplante de Pulmón (TP).

El 90,6\% de la muestra de trasplante hepático (TH) está conformada por varones y el 9,4\% por mujeres. El promedio de edad para este grupo es de 58,22, con un rango que oscila entre 44 y 66 años.

La muestra de Trasplante Pulmonar (TP) está compuesta por un $68,8 \%$ de varones y un $31,3 \%$ de mujeres. La media de edad es de 58,13 , con un rango de 37 a 65 años ${ }^{30}$.

\section{Instrumentos}

Se utilizó un protocolo de valoración elaborado por el equipo de psicología clínica del Servicio de Salud Mental de Enlace e Interconsulta del HUCA. Este protocolo se compuso de los siguientes instrumentos, todos ellos validados para población española:

a. Entrevista Clínica aplicada al candidato a trasplante y a su figura principal de cuidados. Se incluyeron datos sociodemográficos, antecedentes, historia de enfermedad actual, apoyo social percibido, apoyo social disponible y salud mental. Se recogió: exploración psicopatológica, rasgos de personalidad, estrategias de afrontamiento, antecedentes psiquiátricos, estado emocional, estilo de vida, adherencia a tratamientos, necesidades de información y relación con el personal médico, comprensión y expectativas sobre su salud, locus de control e impresión diagnóstica.

b. Escala de Ansiedad y Depresión Hospitalaria (HADS), Zigmond y Snaith, 1983: cuestionario de 14 ítems en formato tipo Likert (0-3) que valora las subescalas de depresión y ansiedad con siete ítems cada una ${ }^{16}$. Según las normas de corrección e interpretación del instrumento, las personas con puntuación de 0 a 7 se sitúan en un rango de normalidad para la subescala; de 8 a 10 como casos probables y de 11 a 21 como casos de ansiedad y depresión.

c. Mini-Examen del Estado Mental (MMSE), Folstein et $\mathrm{al}^{31}$ : cuestionario de cribado de 30 ítems para detectar posible existencia de deterioro cognitivo. Los ítems están agrupados en cinco apartados (orientación espacio-tiempo; atención, concentración y memoria; capacidad de abstracción y cálculo; capacidad de lenguaje y percepción visoespacial y por último capacidad para seguir instrucciones básicas). El punto de corte más ampliamente utilizado se sitúa en 23 puntos. Las puntuaciones iguales o menores a esta cifra indicarían la presencia de un déficit cognoscitivo ${ }^{32}$.

\section{Procedimiento}

Las evaluaciones se desarrollaron en el Servicio de Salud Mental de Enlace e Interconsulta del HUCA y fueron realizadas por cuatro Psicólogas Internas Residentes (PIR) bajo la supervisión de los Facultativos Especialistas en Psicología Clínica de dicho servicio.

Esta evaluación forma parte del Protocolo pretrasplante del hospital que incluye la participación de diferentes especialistas. Desde el Servicio de Salud Mental se emite un juicio clínico a implementar al resto de valoraciones médicas pertinentes para decidir la indicación de trasplante. Cada candidato es valorado de forma individual mediante una entrevista clínica de una duración estimada entre 60 y 90 minutos (incluye también información proporcionada por un cuidador) y la aplicación de las escalas HADS y MMSE.

Para la investigación se seleccionaron todos los candidatos a trasplante hepático y pulmonar derivados por los Servicios de Digestivo y Neumología respectivamente durante el año 2017.

Del volcado de la entrevista a la historia clínica informatizada de cada paciente se recogieron las siguientes variables:

a. Variables sociodemográficas: sexo, edad, estado civil, situación laboral.

b. Variables sociofamiliares: apoyo social percibido. 
c. Estilo de vida saludable y hábitos tóxicos.

d. Estado clínico: presencia de sintomatología ansioso-depresiva.

e. Variables intrínsecas al proceso de trasplante: información y motivación para someterse a la intervención.

\section{Análisis estadístico}

Los datos fueron analizados mediante el programa Statistical Package for the Social Science (SPSS), versión 20.

Se efectúo un análisis descriptivo de la muestra a través de las medias y las desviaciones típicas de las variables de estudio más relevantes. Se realizó a su vez un análisis agrupando a los participantes en función del tipo de trasplante al que eran candidatos.

\section{Declaración sobre aspectos éticos}

Este estudio adaptó las normas establecidas en la Declaración de Helsinki de 1975. Se han tenido en cuenta las recomendaciones para la protección de personas y confidencialidad de los datos, así como el derecho a la privacidad.

En el trabajo y en el momento de su distribución no se incluyeron datos personales, entendiendo por datos personales cualquier información relativa a una persona física identificada o identificable (nombres, iniciales, direcciones, imágenes, analíticas, fotografías, ni números de historias clínicas).

El consentimiento informado se obtuvo de forma verbal, no siendo necesario requerirse por escrito en base a la Ley $41 / 2002$ de 14 de noviembre, básica reguladora de la autonomía del paciente y de derechos y obligaciones en materia de información y documentación clínica ${ }^{33,34}$.

\section{RESULTADOS}

El número de personas derivadas en el año 2017 al programa de valoración psicológica pretrasplante fue de 64 . El 75\% fue valorado para Trasplante de Hígado (TH) y el $25 \%$ restante para valoración de
Trasplante de Pulmón (TP).

A continuación, se presentan los resultados en función de las variables incluidas en el estudio.

\section{Perfil sociofamiliar, apoyo percibido y situación laboral}

El estado civil del $56,3 \%$ de la muestra TH es casado y el $21,9 \%$ separado o divorciado. En la muestra de pacientes del grupo TP, el $50 \%$ de los pacientes está casado y un $25 \%$ soltero.

Respecto al apoyo social percibido, el $81,3 \%$ de los pacientes del grupo $\mathrm{TH}$ reciben un apoyo adecuado frente al $87 \%$ del grupo TP.

En cuanto a la situación laboral, el $62,5 \%$ del total de los sujetos evaluados se encuentra en situación de jubilación o prejubilación.

\section{Estilo de vida saludable y hábitos tóxicos}

Al explorar la percepción de necesidad de mantener hábitos saludables, el $68,8 \%$ de la muestra $\mathrm{TH}$ presenta un nivel alto de conciencia, el 18,8\% relativa conciencia y el $12,5 \%$ restante conciencia limitada. En el grupo TP el 56,3\% presenta conciencia total acerca de la importancia de los hábitos saludables, el $37,5 \%$ muestra conciencia parcial y el $6,3 \%$ escasa o limitada.

Con relación a los hábitos de consumo de tóxicos en el momento de la evaluación, el $43,8 \%$ del grupo TH, es fumador; respecto al consumo de otros tóxicos, un $12,5 \%$ tiene historia previa de consumo (cocaína/heroína). Un $44 \%$ de la muestra consume alcohol o tiene una abstinencia menor a 6 meses (Figura 1). Al analizar los resultados de los candidatos a TP se encuentra que el $43,8 \%$ consume alcohol en la actualidad. El 31,3\% mantiene abstinencia de tabaco inferior a 6 meses y el $12,5 \%$ son fumadores activos.

\section{Estado clínico y alteraciones anímicas asociadas}

Se encuentra que la mitad de los usuarios del grupo TH presenta antecedentes en los Servicios de Salud 
Mental. Durante la entrevista clínica, el 19,7\% presenta indicadores de ansiedad y depresión. Al analizar el grupo TP, se observa que el $62,5 \%$ de los sujetos recibieron asistencia previa en Salud Mental. El $18,7 \%$ de los usuarios muestran indicadores de ansiedad y depresión a través de la entrevista clínica (Figura 2). No obstante, al realizar el cuestionario HADS se registra un leve incremento de los niveles de ansiedad y depresión en ambos grupos de candidatos a trasplante (Figura 3 ).

\section{Variables intrínsecas al proceso de trasplante}

Se observa que menos de la mitad de los pacientes tiene información completa sobre el proceso de trasplante en el momento de la valoración (un 40,6 $\%$ el grupo de $\mathrm{TH}$ y un $37,5 \%$ el grupo de TP). En relación con la motivación para realizar la intervención, los candidatos a TH muestran mayores niveles de motivación (81,3\%), frente al $62,5 \%$ del grupo de TP (Tabla 1).

$\square$ Activo
$\square$ Abstinencia $<6$ meses
$\square$ Abstinencia $6-12$ meses
$\square$ Abstiencia $>12$ meses
$\square$ No consumidor

Figura 1. Consumo de alcohol en pacientes evaluados para trasplante hepático.

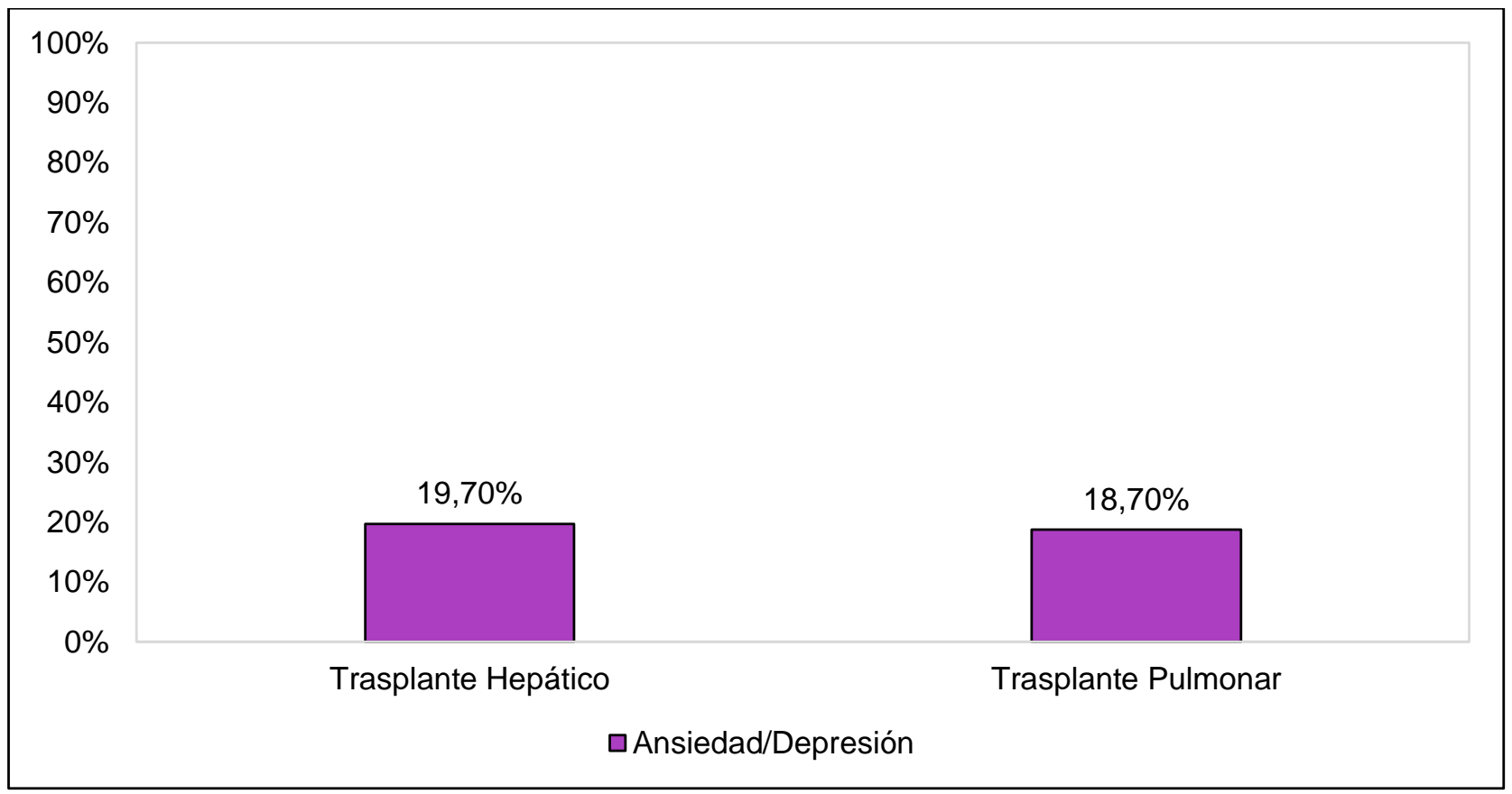

Figura 2. Niveles de ansiedad y depresión medidos a través de la entrevista clínica. 


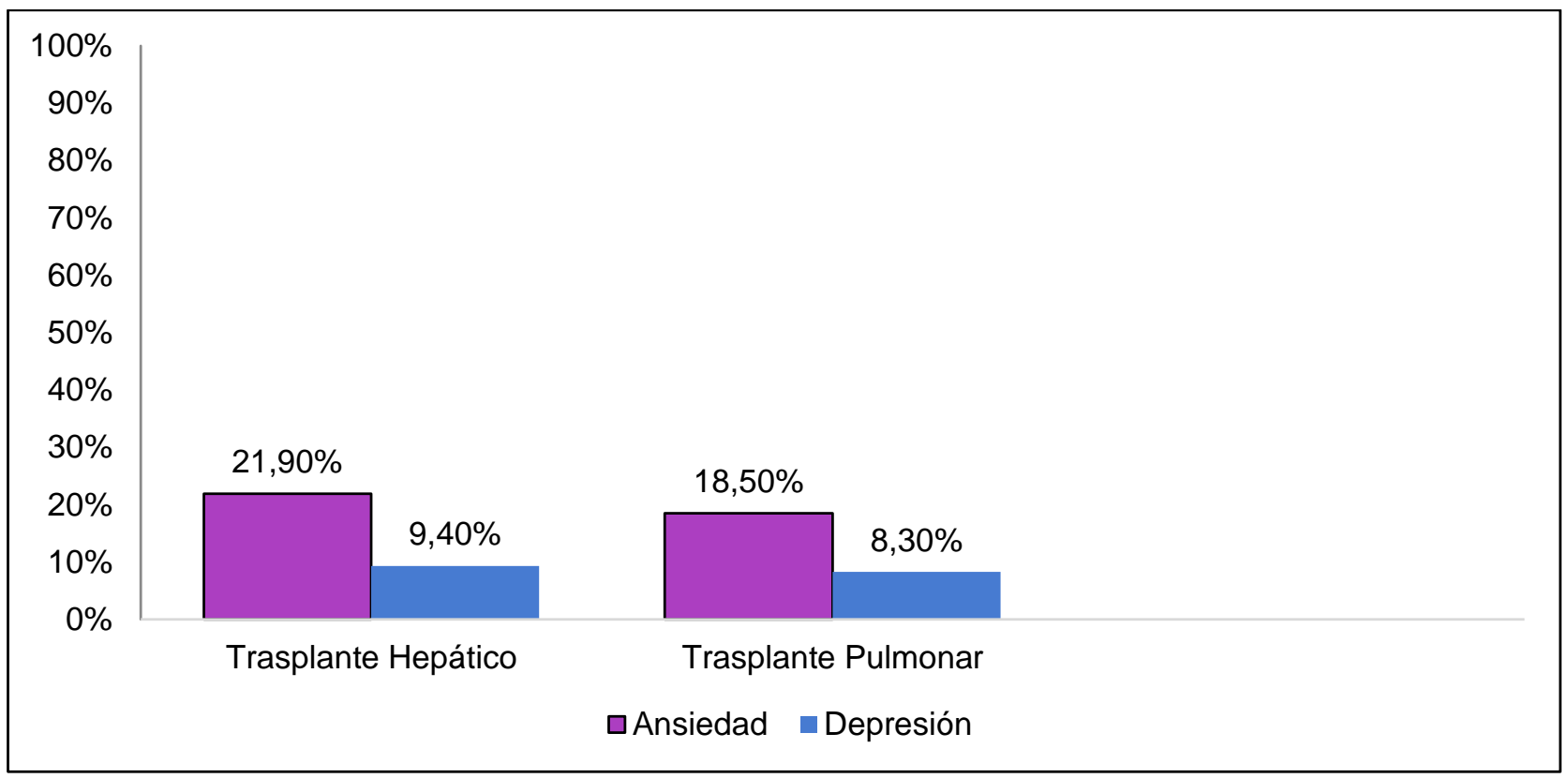

Figura 3. Niveles de ansiedad y depresión medidos a través de la Escala HADS.

Tabla 1. Variables intrínsecas al proceso de trasplante: información recibida y motivación al trasplante.

\begin{tabular}{|c|l|l|l|l|l|l|}
\hline \multirow{2}{*}{ Tipo de trasplante } & \multicolumn{3}{|c|}{ Información recibida } & \multicolumn{3}{c|}{ Motivación al trasplante } \\
\cline { 2 - 7 } & & & & & \\
& Escasa & Parcial & Completa & Escasa & Parcial & Completa \\
\hline Hepático & $\begin{array}{l}37,5 \% \\
(n=12)\end{array}$ & $\begin{array}{l}31,9 \% \\
(n=7)\end{array}$ & $\begin{array}{l}40,6 \% \\
(n=13)\end{array}$ & $\begin{array}{l}6,3 \% \\
(n=2)\end{array}$ & $\begin{array}{l}12,5 \% \\
(n=4)\end{array}$ & $81,3 \%(n=26)$ \\
\hline Pulmón & $\begin{array}{l}12,5 \% \\
(n=4)\end{array}$ & $\begin{array}{l}50 \% \\
(n=16)\end{array}$ & $\begin{array}{l}37,5 \% \\
(n=12)\end{array}$ & $\begin{array}{l}6,3 \% \\
(n=2)\end{array}$ & $\begin{array}{l}31,3 \% \\
(n=10)\end{array}$ & $62,5 \%(n=20)$ \\
\hline
\end{tabular}

\section{DISCUSIÓN}

En relación con el primer objetivo de este estudio, se encuentra que el perfil sociodemográfico de los candidatos a trasplante corresponde a un varón con edad promedio de 60 años, casado y en situación de jubilación o prejubilación. Con relación al apoyo sociofamiliar recibido, la percepción de los entrevistados es adecuada.
Al explorar la necesidad percibida de mantener un estilo de vida saludable (patrones de alimentación y actividad física) y abstinencia de consumos, se encuentran niveles medios de conciencia. Los niveles de conciencia son mayores en los candidatos a trasplante hepático, lo cual puede relacionarse con el momento en que se lleva a cabo la valoración, normalmente en estado avanzado de la enfermedad. Estos pacientes presentan, además, un mayor conocimiento de la necesidad de mantener la 
abstinencia para la evolución favorable del proceso, ya que la intervención quirúrgica suele ser la única alternativa de tratamiento. También se encuentra que un porcentaje significativo de los candidatos a trasplante hepático reconoce no mantener un periodo de abstinencia a alcohol superior a 6 meses, lo cual aumenta notablemente el riesgo de rechazo al órgano postrasplante. Aunque este criterio temporal es uno de los más utilizados, hay otros factores predictores de la abstinencia, como son la comorbilidad psiquiátrica, especialmente la depresión, y tener historia previa de recaída en alcohol $^{19}$.

En este sentido, cabe señalar que el consumo de alcohol en nuestro contexto es una práctica extendida y socialmente aprobada, resultando complicado para los pacientes conseguir una abstinencia total. Además, algunos candidatos no valoran el consumo de ciertas bebidas alcohólicas de menor graduación como un incumplimiento del objetivo de abstinencia. Esto hace necesario realizar valoraciones comprehensivas que completen la información proporcionada por los usuarios en la entrevista clínica, tales como revisión de la historia clínica, analíticas de consumo, información de familiares y coordinación con otros profesionales implicados $^{13,21}$. El abordaje de la conciencia del problema y la motivación para el cambio son aspectos muy relevantes del trabajo terapéutico a lo largo de todo el proceso. Además de los factores pretrasplante, que han sido ampliamente estudiados, también es relevante atender a la abstinencia postrasplante que se ve frecuentemente condicionada por complicaciones posteriores a la intervención, que pueden incrementar el riesgo de recaída, constituyendo un riesgo adicional a las condiciones psicosociales previas $^{13,19}$.

Con referencia al estudio de variables psicopatológicas, llama la atención que, en el momento de la evaluación, a pesar de que más de la mitad de los participantes presenta antecedentes asistenciales previos o actuales en Salud Mental, la mayor parte no informa de sintomatología ansiosa y/o depresiva de relevancia clínica. Estos resultados contrastan con los de otros estudios realizados en este contexto, los cuales encuentran que más de la mitad de los pacientes evaluados para trasplante presentan disfunciones psicológicas ${ }^{28}$.Una posible explicación es que los pacientes y familiares minimizan el malestar emocional debido al sesgo de deseabilidad social y al temor a que pudiera afectar a su oportunidad de ser trasplantados. Consideramos que realizar un escaso número de entrevistas previas puede ser interpretado por ellos como un contexto de valoración más que de ayuda. Por tanto, una evaluación psicológica continuada nos aportaría una visión más fiable y la posibilidad de proporcionar un plan terapéutico durante el proceso pre y postrasplante. En esta línea, existen múltiples investigaciones que avalan la importancia de identificar posibles disfunciones psíquicas. Se recomienda realizar un proceso de evaluación continuada que contemple el estado clínico del paciente tras su inclusión en el programa de trasplante, posteriormente a la intervención y con seguimientos a corto, medio y largo plazo $4,15,23,35,36$. Estudios actuales señalan un aumento de la incidencia de los trastornos del ánimo y ansiedad de hasta un $30 \%$ en pacientes hepáticos, así como de trastorno de estrés postraumático en pacientes que pasan largas hospitalizaciones en Unidades de Cuidados intensivos tras la operación ${ }^{28,35,37}$.

Por último, en lo relativo al propio proceso de trasplante, se observa que la información acerca del mismo es escasa o parcial para ambos grupos. El porcentaje de pacientes que se encuentran motivados para la intervención es superior en los candidatos a trasplante hepático. Esto puede deberse a que son valorados en un momento más avanzado del proceso de enfermedad y su deterioro físico conlleva mayor grado de limitación funcional. La cantidad y la calidad de la información médica recibida sobre el proceso previo, el quirúrgico y el de recuperación posterior, puede influir en la motivación para realizar el trasplante. A su vez, la motivación suele ir acompañada de algunas modificaciones saludables en el estilo de vida. No obstante, se valora que el conocimiento del proceso es necesario, pero no suficiente, especialmente para conseguir mantener el hábito de abstinencia y otros comportamientos saludables que fomenten la adherencia y la consiguiente buena evolución posttrasplante. 
Con base en lo expuesto y en relación con el segundo objetivo de este trabajo, relativo a las contribuciones de la psicología clínica en el trasplante de órganos, es necesario realizar un seguimiento psicológico durante todo el proceso dada su extensa duración, a través de:

a. Evaluaciones de carácter continuado que contemplen los factores psicosociales relevantes en el proceso ${ }^{13}$.

b. Intervenciones específicas de carácter individual o grupal que favorezcan la adherencia, la deshabituación y el mantenimiento de la abstinencia o prevención de recaídas ${ }^{19,38}$.

c. Grupos de apoyo para pacientes y cuidadores que proporcionen información, evitando dinámicas de afrontamiento negativas ${ }^{21,24,40}$, como la conspiración del silencio. Esta hace referencia al acuerdo implícito y/o explícito al que llega la familia, el entorno o los profesionales de la salud, de cambiar u omitir la información brindada al paciente con la finalidad de protegerlo del impacto que pueda tener en su vida saber sobre la situación de su enfermedad ${ }^{39}$.

d. Espacios terapéuticos que permitan la expresión emocional y el abordaje de estrategias para su regulación y un afrontamiento más adaptativo ${ }^{21,23}$.

En definitiva, estas propuestas buscan alejarse del actual marco de evaluación limitado al cribaje y suponen mejoras en la acomodación de la persona a las diferentes fases del proceso, facilitando la atención integral y pudiendo contribuir al desarrollo exitoso del mismo.

\section{AGRADECIMIENTOS}

Agradecimientos al Servicio de Salud Mental de Enlace e Interconsulta del Hospital Universitario Central de Asturias y a la Directora de Gestión Clínica del área IV del SESPA por posibilitar y facilitar la realización de este trabajo durante nuestra etapa de residentes de Psicología Clínica.

\section{DECLARACIÓN SOBRE CONFLICTO DE INTERESES}

Los autores declaran que no existe conflicto de intereses.

\section{CONTRIBUCIÓN DE LOS AUTORES}

Primera autora: trabajo de campo y redacción;

Segunda autora: redacción y análisis estadístico;

Tercera autora: diseño metodológico y análisis estadístico;

Cuarta autora: diseño metodológico y trabajo de campo.

\section{REFERENCIAS BIBLIOGRÁFICAS}

1. Burgos VA, Amador A, Ballesteros BP. Calidad de vida en pacientes con trasplante de hígado. Univ Psychol Bogotá [Internet]. 2007 [Consultado 5 Jul 2019]; 6(2): 383-97. Disponible en: http://pepsic.bvsalud.org/pdf/up/v6n2/v6n2a16.p df

2. Costa Requena G, Rodriguez Urrutia A, Parramon G. Interconsulta y psiquiatría de enlace: estudio descriptivo de las interconsultas a psicología clínica. C Med Psicosom [Internet]. 2015 [Consultado 4 May 2019]; 115: 11-5. Disponible en: https://dialnet.unirioja.es/servlet/articulo?codigo= 5208939

3. Daga-Ruiz D, Fernández Aguirre C, Segura González F, Carballo Ruiz M. Indicaciones y resultados a largo plazo de los trasplantes de órganos sólidos. Calidad de vida en pacientes trasplantados. Med Intesiva [Internet]. 2008 [Consultado 6 May 2019]; 32(6): 296-303. Doi: https://doi.org/10.1016/S0210-5691(08)70957-4

4. García-Flores A. Evaluación psicológica y social del paciente receptor de trasplante renal. Abordaje psicosocial del postrasplante renal. RevMexTraspl [Internet]. 2014 [Consultado 4 May 2019]; 3(3): 95- 
$101 . \quad$ Disponible en:

http://www.medigraphic.com/trasplantes

5. Organización Nacional de Trasplantes. Memoria ONT 2016 [sede Web]; Ministerio de Sanidad, Servicios Sociales e Igualdad. Disponible en: http://www.ont.es/Paginas/Home.aspx

6. Octavio-Rojas G, Vania-Krauskopf P, AgustínUmaña MJ, Rodrigo-Erazo R. Intervención psiquiátrica en programa de trasplantes. Rev Med Clin Condes [Internet]. 2010 [Consultado 23 Jun 2019]; 21(2): 86-292. Disponible en: http://www.clcmovil.cl/Dev_CLC/media/Imagenes/ PDF\%20revista\%20m\%C3\%A9dica/2012/5

\%20sept/13_Dr--Octavio-Rojas-G.-6.pdf

7. Matesanz R. El modelo español de coordinación y trasplantes. 2a ed. Madrid: Grupo Aula Médica. $2008 . \quad$ Disponible en: http://www.ont.es/publicaciones/Documents/mod eloespanol.pdf

8. Vinaccia S, Orozco LM. Aspectos psicosociales asociados con la calidad de vida de personas con enfermedades crónicas. Diver Perspet Psicol [Internet]. 2005 [Consultado 6 May 2019]; 1(2): 125$37 . \quad$ Disponible en: https://www.redalyc.org/pdf/679/67910202.pdf

9. Engle D. Psychosocial aspects of the organ trasplant experience: what has been stablished and what we need for the future. J Clin Psychol [Internet]. 2001[Consultado 22 Mar 2018]; 57(4):521-49.

Doi: https://doi.org/10.1002/jclp.1027

10. Corbett C, Amstrong M, Parker R, Webb K, Neuberger J. Mental health disorders and solid organ. Transplantation [Internet]. 2013 [Consultado 2 Jun 2019]; 96: 593-600. Doi: http://doi.org/10.1097/TP.0b013e31829584e0

11. Messias E, Skotzko C. Psychiatric assessment in transplantation. Rev Saúde Pública [Internet]. 2000 [Consultado 23 Jun 2019]; 34(4): 415-2. Doi: http://dx.doi.org/10.1590/\$0034-

89102000000400018
12. Kern de Castro E, Moreno-Jiménez, B. Competencia social y problemas emocionales/conductuales en niños trasplantados de órganos sólidos. Psicología Conductual [Internet]. 2008 [Consultado 28 Jun 2019]; 16(2): 307-20. Disponible en: https://dialnet.unirioja.es/servlet/articulo?codigo= 2699299

13. Akyüz-Ózdemir A, Burak-Sayin C, ErdalR,Ózcan C, Haberal M. Influence social, economic,familial, marital status, disease adaptation on the physical and mental health dimensions who are candidants for renal transplant. Experimental and Clinical Transplantation [Internet]. 2018 [Consultado 3 May 2019]; 1: 112-6. Doi: https://doi.org/10.6002/ect.tond-tdtd2017.p4

14. Sher Y, Maldonado JR. Psychosocial care of endstage organ disease and transplant patients.1eed. Stanford: Springer; $2019 . \quad$ Doi: https:doi.org/10.1007/978-3-319-94914-7

15. Hitschfeld $M$, Schneekloth $T$, Kennedy $C$, Rummans T, Niazi $S$, Vasquez $A$ et al. The psichosocial assessment of candidates for trasplantation: a cohort study of its association with survival among lung transplant recipients. Psychosomatics [Internet]. 2016 [Consultado 3 May 2019]; 57 (5): 489-97. Doi: https://doi.org/10.1016/j.psym.2016.05.003

16. Collins C, Labott S. Psychological assessment of candidates for solid organ transplantation. Professional Psychology: Research and Practice [Internet]. 2007 [Consultado 6 May 2019]; 38(2): 150-7. Doi: https://doi.org/10.1037/07357028.38.2.150

17. López Fuentetaja AM, Iriondo Villaverde $\mathrm{O}$. Intervención psicológica en el ámbito hospitalario. Revista Clínica Contemporánea [Internet]. 2019 [Consultado 8 Jul 2019]; 10: 1-19. Doi: https://doi.org/10.5093/cc2019a2

18. Levinson JL, Olbrish ME. Psychosocial evaluation of organ transplant candidates. Psychosomatics [Internet]. 1993 [Consultado 2 Ene 2019] ; 34:31423. Doi: https://doi.org/10.1016/S00333182(93)71865-4 
19. Kitajima T, Nagai S, Segal A, Magee M, Blackburn $S$, Ellithorpe $D$ et al. Posttrasplant complications predict alcohols relapse in liver transplant recipients. Liver Trasplantantion [Internet]. 2020 [Consultado 8 Jun 2019]; 6(3): 379-89. Doi: https://doi.org/10.1002/It.25712

20. Coffman KL, Siemionow MZ. Face transplantation: psychological outcomes at three year follow up. Psychosomatics [Internet]. 2013 [Consultado 6 May 2019]; 54: 372-8. Doi: https://doi.org/10.1016/j.psym.2012.10.009

21. Rodríguez T, Fonseca Fernández M. Guía de atención psicológica a pacientes y familiares en el proceso de donación y trasplante de órganos. Medisur [Internet]. 2015 [Consultado 8 Jun 2019]; 13(4): 560-8. Disponible en: http://www.medisur.sld.cu/index.php/medisur/arti cle/view/3029

22. Mena-Nájera A, Barragán-Fuentes AG, Gómezde-Regil L. Intervenciones psicológicas en pacientes con trasplante renal. Evid Med Invest Salud [Internet]. 2014 [Consultado 6 May 2019]; 7(2): 70$5 . \quad$ Disponible en: https://www.medigraphic.com/pdfs/evidencia/eo2014/eo142d.pdf

23. Bamford J, Wirz L. Piloting psychology annual reviews as a method of measuring psychological distress and quality of life in pediatric renal transplant patients. BioMed Research International [Internet]. 2016 [Consultado 17 Mar 2019]; 2016: 09. Doi: https://doi.org/10.1155/2016/1685362

24. Ok Soo K, Kyung Sook C. Influence of family and medical staff support and hope on organ transplant recipients' psychosocial adjustment. JKCNR [Internet]. 2016 [Consultado 17 Mar 2019]; 22(1): 78-87.

Doi: https://doi.org/10.22650/JKCNR.2016.22.1.78

25. Pérez MA, Rodríguez AM, Galán A. Problemas psicológicos asociados al trasplante de órganos. Int $\mathrm{J}$ Clin Health Psychol [Internet]. 2005 [Consultado 24 Mar 2019]; 5(1): 99-114. Disponible en: https://www.redalyc.org/pdf/337/33701006.pdf
26. Herrero MJ, Blanch JM, Peri J, De Pablo L, Bulbena A. A validation study of the hospital anxiety and depression scale (HADS) in a Spanish population. General Hospital Psychiatry [Internet]. 2003 [Consultado 24 Mar 2019]; 25(4): 277-83. Doi: https://doi.org/10.1016/S0163-8343(03)00043-4

27. Taylor J, Smith P, Babyak M, Barbour K, Hoffman $B$, Sebring $D$ et al. Coping and quality of life in patients awaiting lung transplantation. J Psychosom Res [Internet]. 2008 [Consultado 6 May 2019]; 65(1): 71-9.

Doi:

https://doi.org/10.1016/j.jpsychores.2008.04.009

28. Randall J, Kayser K. Pre-Transplant Psychosocial Assessment practices: a national survey of transplant centers. Biology of Blood and Marrow Transplantation [Internet]. 2020 [Consultado $13 \mathrm{Jul}$ 2020]; 26(3): S204. Doi: https://doi.org/10.1016/j.bbmt.2019.12.698

29. Ato M, López JJ, Benavente A. Un sistema de clasificación de los diseños de investigación en psicosocial. Anales de Psicología [Internet]. 2013 [Consultado 17 May 2019]; 9(3): 1038-59. Doi: https://doi.org/10.6018/analesps.29.3.178511

30. Otzen T, Manterola C. Técnicas de muestreo sobre una población a estudio. Int J Morphol [Internet]. 2017 [Consultado 20 May 2019]; 35(1): 227-32. Doi: http://dx.doi.org/10.4067/S071795022017000100037

31. Folstein MF, Folstein SE, McHugh PR. "Mini Mental State". A practical method for granding the cognitive state of patients for de clinician. J. Psychiatr [Internet]. 1975 [Consultado 23 May 2019]; 12(3):189-98. Doi: https://doi.org/10.1016/0022-3956(75)90026-6

32. López Miguel J, Martí Agustí G. Mini-Examen Cognoscitivo (MEC). Rev Esp Med Legal [Internet]. 2011 [Consultado 6 May 2019]; 37(3):122-7. Doi: https://doi.org/10.1016/S0377-4732(11)70075-X

33. Ley $41 / 2002$, de 14 de noviembre, básica reguladora de la autonomía del paciente y de derechos y obligaciones en materia de información 
y documentación clínica [en línea]. Madrid: Boletin Oficial del Estado;2002 [citado el 14 julio de 2020]. Disponible en:

https://www.boe.es/eli/es/l/2002/11/14/41

34. García-Ortega C, Cózar-Murillo V, AlmenaraBarrios J. La autonomía del paciente y los derechos en materia de información y documentación clínica en el contexto de la Ley 41/2002. Rev Esp Salud Pública [Internet]. 2004 [Consultado 20 May 2019]; 78: 469-79. Disponible en: http://scielo.isciii.es/scielo.php?script=sci_arttext\& pid=S1135-57272004000400005\&lng=es

35. Paterson $T, O^{\prime}$ Rourke $N$, Shapiro $R$, Loken Thornton $W$. Medication adherence in renal transplant recipients: a latent variable model of psychosocial and neurocognitive predictors. PLOS ONE [Internet]. 2018 [Consultado 13 Mar 2019]; 13(9): e0204219. Doi: https://doi.org/10.1371/journal.pone.0204219

36. Magán-Uceda I, Cuellar-Roche B, López-Sánchez V, Segade-Álvarez M, Dávila-Madrigal F, GarcíaAcero $\mathrm{C}$ et al. Conviviendo con la incertidumbre: intervención psicológica en una mujer con un problema de adaptación en lista de espera para trasplante de pulmón. Clínica y Salud [Internet]. 2015 [Consultado 13 Jul 2019]; 26(3): 151-8. Doi: https://doi.org/10.1016/j.clysa.2015.09.002

37. Krauskopf V. Evaluación psiquiátrica en trasplantes. Rev Med Clin Condes [Internet]. 2017 [Consultado 16 May 2019]; 28(6): 849-54. Doi: https://doi.org/10.1016/j.rmclc.2017.11.006
38. Chávez-Victorino O. Efectos de una intervención psicológica sobre la adhesión terapéutica en pacientes con insuficiencia renal en espera de trasplante renal. Rev Elec de Psiclz [Internet]. 2018 [Consultado 6 May 2019]; 21(1): 199-221. Disponible en: https://www.medigraphic.com/pdfs/epsicologia/e pi-2018/epi181k.pdf

39. Espinoza-Suárez NR, Zapata del Mar CM, MejíaPerez LA. Conspiración del silencio: una barrera en la comunicación médico, paciente y familia. Rev Neuropsiquiatr [Internet]. 2017 [Consultado 6 May 2019]; 8(2): 125-36. Doi: https://doi.org/10.20453/rnp.v80i2.3105

40. Jesse MT, Shkokani L, Eshelman A, De Reyck C, Abouljoud $M$, Lerut J. Transplant surgeon burnout and marital distress in the sandwich generation: the call for organizational support in family life. Transplantation Proceedings [Internet]. 2018 [Consultado 20 Mar 2019]; 50(1): 2899-904. Doi: https://doi.org/10.1016/j.transproceed.2018.01.05 3 